\title{
Pulmonary Toxicity in a Renal Transplant Recipient Treated with Amiodarone and Everolimus: A Case of Hypothetical Synergy and a Proposal for a Screening Protocol
}

\author{
Alberto Mella ${ }^{a} \quad$ Maria Messina $^{a} \quad$ Andrea Ranghino $^{a}$ Paolo Solidoro $^{b}$ \\ Giuseppe Tabbiab ${ }^{b}$ Giuseppe Paolo Segoloni ${ }^{a}$ Luigi Biancone $^{a}$ \\ ${ }^{a}$ Renal Transplantation Unit 'A. Vercellone', Division of Nephrology Dialysis and \\ Transplantation, Department of Medical Sciences, and ${ }^{b}$ Lung Disease Unit, Cardiovascular \\ and Thoracic Department, AOU Città della Salute e della Scienza Hospital, University of \\ Turin, Turin, Italy
}

\section{Key Words}

Amiodarone - Everolimus · Pulmonary toxicity $\cdot$ Renal transplant

\begin{abstract}
Pneumotoxic drugs like amiodarone and m-TOR inhibitors (m-TORi) may be administered contemporaneously in therapy for patients who had renal transplants. We present a case of amiodarone pulmonary toxicity (APT) in a patient treated with amiodarone and everolimus. A 57-year-old Caucasian male, under treatment with both everolimus (for 3 years) and amiodarone (for 2 months), presented with fever, dyspnoea and a negative chest X-ray after his second kidney transplant with suboptimal serum creatinine $(3 \mathrm{mg} / \mathrm{dl})$. A non-contrastive high-resolution CT scan showed bilateral interstitial lung disease with an associated reduction in carbon monoxide diffusing capacity. Bronchoalveolar lavage (BAL) was negative for an infection, but BAL cytology was suitable for APT ( $50 \%$ of 'foamy' macrophages). A complete recovery was achieved after amiodarone interruption and an oral steroid therapy increase. Everolimus was continued. His kidney function remained unchanged in the upcoming months. In conclusion, we suggest a possible synergistic effect between m-TORi and amiodarone. Furthermore, we propose a diagnostic algorithm that can be used as a surveillance tool to identify a potential initial lung damage in patients treated with 1 or more pneumotoxic drugs.




\section{Introduction}

Amiodarone, a benzofuran derivative, is one of the most widely used anti-arrhythmic agents in renal transplant patients [1]. Unfortunately, despite its undoubted effectiveness, amiodarone has many side effects, including corneal micro deposits, optic neuropathy and/or optic neuritis, photosensitivity, blue-gray pigmentation, hypo- and hyperthyroidism, hepatic disorders, and amiodarone pulmonary toxicity (APT) [2].

APT occurs in approximately $5 \%$ of treated patients and represents the most serious and life-threatening side effects (mortality ranges from $9 \%$ in chronic pneumonia to $50 \%$ in patients who develop acute respiratory distress syndrome) [3].

Several other drugs can be responsible for pulmonary toxicity. The inhibitors of the mammalian target of rapamycin (mTORi) were approved in 1999 as immunosuppressants for the use in transplants. Since 2000, some cases of non-infectious pneumonitis have been reported in solid organ transplants in which the patients were treated with mTORi [4].

Herein, we present a case of APT in a renal transplant recipient treated with amiodarone and everolimus.

\section{Case Report}

A 57-year-old Caucasian male with end-stage renal disease, secondary to chronic glomerulonephritis (not biopsy proven, but suggested by the evidence of a nephrotic range proteinuria), received a second renal transplant in our center in January 2004. He was initially treated with tacrolimus (Prograf ${ }^{\circledR}$, Astellas Pharma), steroids, mycophenolate mofetil (CellCept ${ }^{\circledR}$, Roche), and then replaced by everolimus (Certican ${ }^{\circledR}$, Novartis Pharma) in 2008 after a biopsy demonstrated chronic allograft nephropathy and severe arteriosclerosis.

The patient's medical history included chronic microcytic hypochromic anemia (hemoglobin $10-10.4 \mathrm{~g} / \mathrm{dl}$ ), hepatitis $\mathrm{C}$ viremia, mild hypertension, hyperlipidemia with intolerance to statin therapy with myalgia and creatine phosphokinase increase.

In October 2011, amiodarone (initial dose $600 \mathrm{mg}$ /day, maintenance dose $200 \mathrm{mg} /$ day), and warfarin were started because of a paroxysmal atrial fibrillation. At this time, echocardiography showed a normal ejective fraction (65\%) with a moderate left ventricular hypertrophy and a severe left atrial dilation (estimated volume $180 \mathrm{ml}$ ) with no valvular diseases.

In December 2011, he presented with malaise, shortness of breath, fever, dyspnoea with minimal effort and worsening of renal function (serum creatinine up to $4.3 \mathrm{mg} / \mathrm{dl}$ ).

On presentation, he was afebrile with normal blood pressure and oxygen saturation. Arterial blood pressure was $123 / 72 \mathrm{~mm}$ Hg. The physical examination was notable only for decreased breathing sounds, but without any chest X-ray alteration. The blood tests showed an isolated increase of C-reactive protein $(155 \mathrm{mg} / \mathrm{l})$ with a normal white blood cell count. Hemoglobin, cholesterol, and triglycerides were unchanged $(9.8 \mathrm{~g} / \mathrm{dl}, 272$ and $153 \mathrm{mg} / \mathrm{dl}$, respectively). Daily immunosuppressive therapy on admission included low-dosage tacrolimus (3 mg/day with target levels $2-3 \mathrm{ng} / \mathrm{ml})$, everolimus $(1.75 \mathrm{mg} /$ day with target levels $5-6 \mathrm{ng} / \mathrm{ml}$ ), and prednisone (5 mg/day). Other medication was irbesartan, doxazosin, amlodipine, metoprolol, omeprazole, furosemide, allopurinol, long-chain omega-3 fatty acids, magnesium pidolate, ferrous liposomes, warfarin, and amiodarone.

Early in hospitalization, the patient became febrile and his respiratory symptoms became relevant. No improvement was noted in spite of his antibiotic treatment (ceftriaxone, piperacillin-tazobactam and doxycycline). On day 10, a high-resolution CT scan (HRCT) of the chest showed a scattered, bilateral, interstitial lung disease. Neither clinical nor 
radiological signs of pulmonary oedema were present. Everolimus levels were within the normal range. No fluid overload was noted, so the dose of diuretics was not increased, and urine output was unchanged. The echocardiographic pattern was unmodified. Pulmonary function tests (PFTs) revealed moderate air trapping and decreased values of carbon monoxide diffusing capacity (DLCO).

A bronchoscopy with bronchoalveolar lavage (BAL) was performed on day 15. During the procedure, no macroscopic alteration and/or sign of pulmonary oedema was noted. BAL did not identify any bacterial, fungal or viral organisms, but BAL cytology detected 'foamy' macrophages (50\% of macrophages counted with a normal total cell count). On day 17 , amiodarone was stopped, and oral steroid therapy was increased from 5 to $12.5 \mathrm{mg} /$ day. Everolimus was maintained with careful level monitoring. Gradually, his pulmonary and clinical status improved, the patient became afebrile, and renal function improved.

On day 30, the patient was discharged. In March 2012, PFTs showed an improvement in DLCO and the steroid dose was reduced from 12.5 to $7.5 \mathrm{mg} /$ day. A further reduction to 5 mg was initiated in April when HRTC was repeated and demonstrated a complete disappearance of the bilateral interstitial disease.

After his discharge, the pulmonary symptoms were absent. The patient's renal function was unchanged (serum creatinine between 3 and $3.5 \mathrm{mg} / \mathrm{dl}$ ), maintaining the same immunosuppressive therapy with everolimus, low-dose tacrolimus and steroids.

\section{Discussion}

Amiodarone, because of its high level of effectiveness, is used worldwide, notwithstanding amiodarone-related adverse effects. APT, the most serious and life threatening complication, occurs in $5-10 \%$ of patients [3].

APT may also sporadically start at the beginning of therapy, even after its withdrawal, without a threshold dose, but it has been commonly described in patients treated with an amiodarone dose between 300 and $800 \mathrm{mg} /$ day. Doses greater than $400 \mathrm{mg} /$ day should be avoided. The onset of pulmonary toxicity may either be insidious or rapidly progressive, with a wide range of clinical presentations, which include acute lung injury (from fibrinous organising pneumonia to acute respiratory distress syndrome) and subacute/chronic patterns (chronic organising pneumonia, lung fibrosis). Pleural/pericardial involvement can be observed.

Three different mechanisms of lung toxicity have been suggested: cytotoxic, angiotensin-related and immune-mediated lung toxicity [5] (fig. 1).

Clinical symptoms like cough, shortness of breath, dyspnoea, new chest infiltrates in imaging studies, and a reduction in DLCO are the cardinal elements for a diagnosis; a meticulous exclusion of infection, malignancy and pulmonary oedema has to be made first [6].

In this context, BAL has a key role, providing evidence of APT (with 'foamy' macrophages on cytology). However, in mTORi patients, BAL could also help diagnosing mTORi lung toxicity by demonstrating a different pattern of cytology (lymphocytic or eosinophilic alveolitis). The role of a lung biopsy is under debate, but at the present time, lung biopsy is considered only when the aforementioned tests are inconclusive. The histological pattern is normally characterized by intra-alveolar foamy macrophages in APT [7] and by a lymphocytic interstitial infiltrate in mTORi lung toxicity $[8,9]$.

In our case, some data are unusual. The symptoms of pulmonary disfunction were relevant, despite a scattered interstitial lung disease. The oral amiodarone initial dose was low, 
as well as the maintenance regimen dose; moreover, well-known risk factors (older age, preexisting lung disease or a history of cardiothoracic surgery) were absent [10]. Besides, the patient had long been treated with angiotensin converting enzyme inhibitors (albeit withdrawn 1 month before hospitalisation) and angiotensin receptor blockers, which are the only 2 drugs that may have a somewhat 'protective role' towards APT [11].

In the authors' opinion, a synergistic effect between mTORi and amiodarone could be hypothesised. We suggest a possible connection between m-TORi pulmonary toxicity and APT (fig. 2).

It has been postulated that lysosomal phospolipid accumulation, causing a formation of 'foamy' macrophages (first step in the APT causative mechanism) [7], results in lysosomal disfunction with an increasing autophagy. Activation of the mTORC1 complex is known to reduce autophagy and, hence, the immunosuppressive drugs are able to inhibit the mTOR complex and could controversely produce a worsening of the pulmonary amiodarone side effects by increasing lysosomal-induced autophagy [12]. In our opinion, this mechanism, first reported in a patient treated with sirolimus and pentamidine [13], could also possibly occur when other drugs - able to induce phospholipid accumulation (such as amiodarone) and mTORi - are prescribed together, facilitating the development of APT. The mutual situation is not applicable because m-TORi pulmonary toxicity seems to be triggered by a different stimulus and has a different immunological pathway [8].

To the best of our knowledge, 2 cases of interstitial pneumonia in transplanted patients, treated with an mTORi (sirolimus and everolimus, respectively) and amiodarone, have been reported [14, 15]. In both papers, patients ceased both amiodarone and mTORi in a condition of pulmonary interstitial damage, with a suspected cumulative toxicity (patient No. 2 in Haydar et al.'s [14] paper and patient No. 2 in Otton et al.'s [15] paper). This issue is not commented on in the paper by Otton et al. [15], while Haydar et al. [14] suggested the hypothesis of a potential synergistic effect. Besides, when everolimus was resumed [15], pneumonia relapsed, too. There is no mention of amiodarone reintroduction. In the case described by Haydar et al. [14], neither amiodarone nor sirolimus were restarted, but during the development of interstitial pneumonitis BAL, the cytology was characterized by an abundance of lymphocytes. Based on these data, one could assume lung damage to be mTORi-induced in both cases.

In our patient, on the contrary, we indicate amiodarone toxicity as the culprit of the interstitial pneumonia (in a patient also taking everolimus). After amiodarone withdrawal, lung pathology regressed while everolimus therapy continued. To the best of our knowledge, this is the first case reported in the literature. We are aware that this synergistic toxicity is not demonstrated in this single case, but it is deducible based on the literature data regarding the mechanism of damage caused by the 2 drugs.

According to our experience, as a first step, we suggest that amiodarone is first prescribed for a patient who is taking m-TORi or vice versa. PFTs, DLCO tests, chest X-rays, and non-invasive lung screening tests are performed as baseline treatments. In case of initial symptoms of lung disease, an in-depth analysis (HRCT, BAL) should be promptly performed. After the diagnosis of drug pulmonary toxicity, treatment is composed by: drug interruption, steroid treatment, repetition of PFTs after 1 month, and HRCT after 2 months. Steroid dose and treatment duration are very individual and based on the severity of the disease and the patients' comorbities; as a first line treatment, steroids are used at a $0.8-1 \mathrm{mg} / \mathrm{kg}$ dose for 34 weeks, with tapering doses of $10 \mathrm{mg} /$ day.

Moreover and as a second step, we propose a diagnostic algorithm which is used as a surveillance tool and is able to predict initial lung damage in a patient treated with mTORi and/or other pneumotoxic drugs (fig. 3). 
Mella et al.: Pulmonary Toxicity in a Renal Transplant Recipient Treated with Amiodarone and Everolimus: A Case of Hypothetical Synergy and a Proposal for a Screening Protocol

As described in figure 3, a DLCO test could be performed before starting the potential pneumotoxic drug and repeated 6 months after. If the difference between the 2 tests is greater than $10 \%$, we suggest performing HRTC, even in case of an interstitial pneumonitis diagnosis (BAL). Otherwise, DLCO should be repeated after 6 months. With a difference between 5 and $10 \%$ on DLCO, a further evaluation should be planned after 1 month; with a less than 5\% difference, we suggest repeating the DLCO test after 6 months.

In conclusion, in patients with a fragile hemodynamic and immunological balance as renal transplant recipients, the contemporary use of pneumotoxic drugs requires careful monitoring and thorough follow-up in view of a possible synergistic mechanism in lung toxicity.

\section{Disclosure Statement}

The authors declare no conflict of interest.

\section{References}

1 Lentine KL, Schnitzler MA, Abbott KC, Li L, Xiao H, Burroughs TE, Takemoto SK, Willoughby LM, Gavard JA, Brennan DC: Incidence, predictors, and associated outcomes of atrial fibrillation after kidney transplantation. Clin J Am Soc Nephrol 2006;1:288-296.

$\longrightarrow 2$ Vassallo P, Trohman RG: Prescribing amiodarone: an evidence-based review of clinical indications. JAMA 2007;298:1312-1322.

-3 Ernawati DK, Stafford L, Hughes JD: Amiodarone induced pulmonary toxicity. Br J Clin Pharmacol 2008;66:82-87.

4 Morelon E, Stern M, Kreis H: Interstitial pneumonitis associated with sirolimus therapy in renal-transplant recipients. N Engl J Med 2000;343:225-226.

5 Ott MC, Khoor A, Leventhal JP, Paterick TE, Burger CD: Pulmonary toxicity in patients receiving low-dose amiodarone. Chest 2003;123:646-651.

6 Goldschlager N, Epstein AE, Naccarelli GV, Olshansky B, Singh B, Collard HR, Murphy E, Practice Guidelines Sub-committee, North American Society of Pacing and Electrophysiology (HRS): A practical guide for clinicians who treat patients with amiodarone: 2007. Heart Rhythm 2007;4:1250-1259.

7 Bedrossian C, Warren C, Ohar J, Bhan R: Amiodarone pulmonary toxicity: cytopathology, ultrastructure, and immunocytochemistry. Ann Dign Pathol 1997;1:47-56.

-8 Exposito V, De Prada JA, Gomez-Roman JJ, Gonzalez-Vilchez F, Llano-Cardenal M, Garcia-Camarero T, Fernandez-Valls M, Ruano J, Martin-Duran R: Everolimus-related pulmonary toxicity in heart transplant recipients. J Heart Lung Transplant 2008;27:797-800.

-9 Morelon E, Stern M, Israel-Biet D, Correas JM, Danel C, Mamzer-Bruneel MF, Peraldi MN, Kreis H: Characteristics of sirolimus-associated interstitial pneumonitis in renal transplant patients. Transplantation 2011;72:787-790.

10 Yamada Y, Shiga T, Matsuda N, Hagiwara N, Kasanuki H: Incidence and predictors of pulmonary toxicity in Japanese patients receiving low-dose amiodarone. Circ J 2007;71:1610-1616.

11 Kosseifi SG, Halawa A, Bailey B, Micklewright M, Roy TM, Byrd RP Jr: Reduction of amiodarone pulmonary toxicity in patients treated with angiotensin-converting enzyme inhibitors and angiotensin receptor blocker. Ther Adv Respir Dis 2009;3:289-294.

12 Anderson N, Borlak J: Drug-induced phospholipidosis. FEBS Lett 2006;580:5533-5540.

-13 Filippone EJ, Carson JM, Beckforf RA, Jaffe BC, Newman E, Awsare BK, Doria C, Farber JL: Sirolimus-induced pneumonitis complicated by pentamidine-induced phospholipidosis in a renal transplant recipient: a case report. Transplantation Proc 2011;43:2792-2797.

14 Haydar AA, Denton M, West A, Rees J, Goldsmith DJ: Sirolimus-induced pneumonitis: three cases and a review of the literature. Am J Transplant 2004;4:137-139.

15 Otton J, Hayward CS, Keogh AM, Glanville AR, Macdonald PS: Everolimus-associated pneumonitis in 3 heart transplant recipients. J Heart Lung Transplant 2009;28:104-106. 
Mella et al.: Pulmonary Toxicity in a Renal Transplant Recipient Treated with Amiodarone and Everolimus: A Case of Hypothetical Synergy and a Proposal for a Screening Protocol

Alberto Mella and Maria Messina contributed equally to this work.

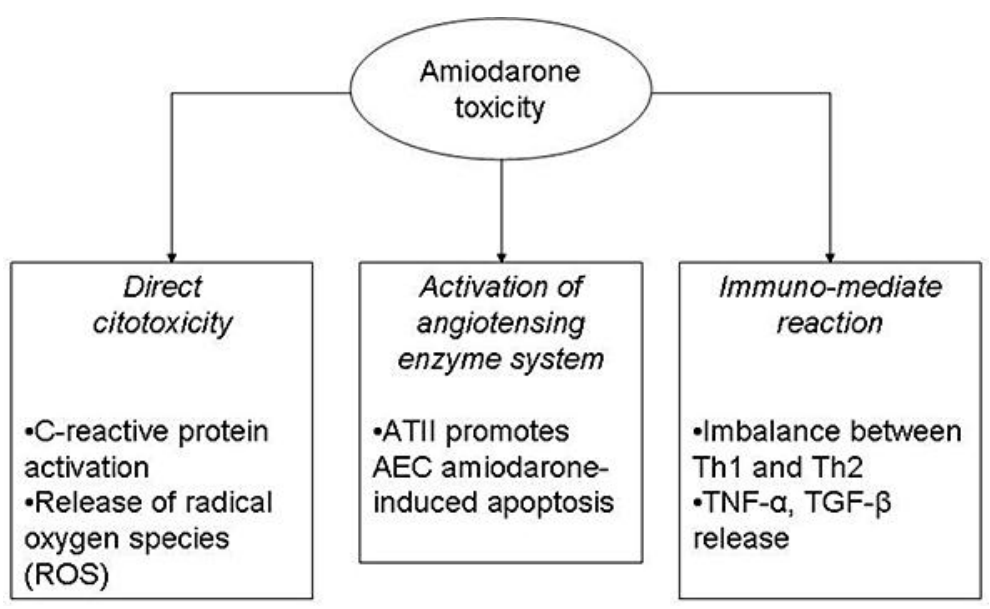

Fig. 1. Amiodarone mechanisms of lung toxicity. $\mathrm{AEC}=$ Alveolar epithelial cells; $\mathrm{Th} 1=\mathrm{T}$ helper type 1 lymphocyte; Th $2=$ T helper type 2 lymphocyte; TNF- $\alpha=$ tumour necrosis factor $-\alpha$; TGF- $\beta=$ transforming growth factor- $\beta$.

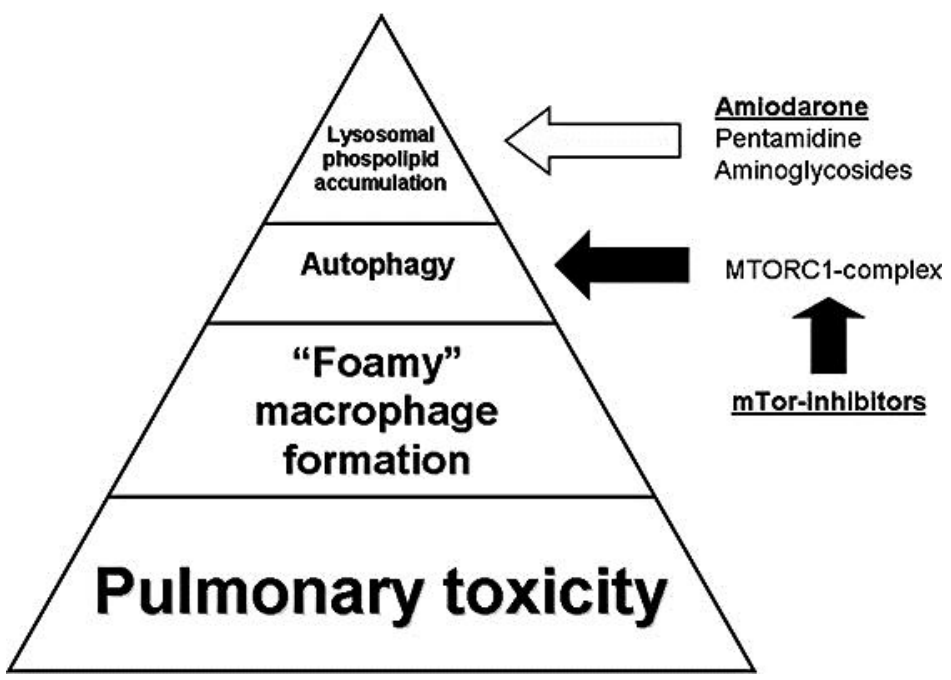

Fig. 2. Scheme for a hypothetical synergistic effect between m-TORi and amiodarone in lung toxicity. Promoting effect: white arrows; inhibiting effect: black arrows. 
Mella et al.: Pulmonary Toxicity in a Renal Transplant Recipient Treated with

Amiodarone and Everolimus: A Case of Hypothetical Synergy and a Proposal for a Screening Protocol

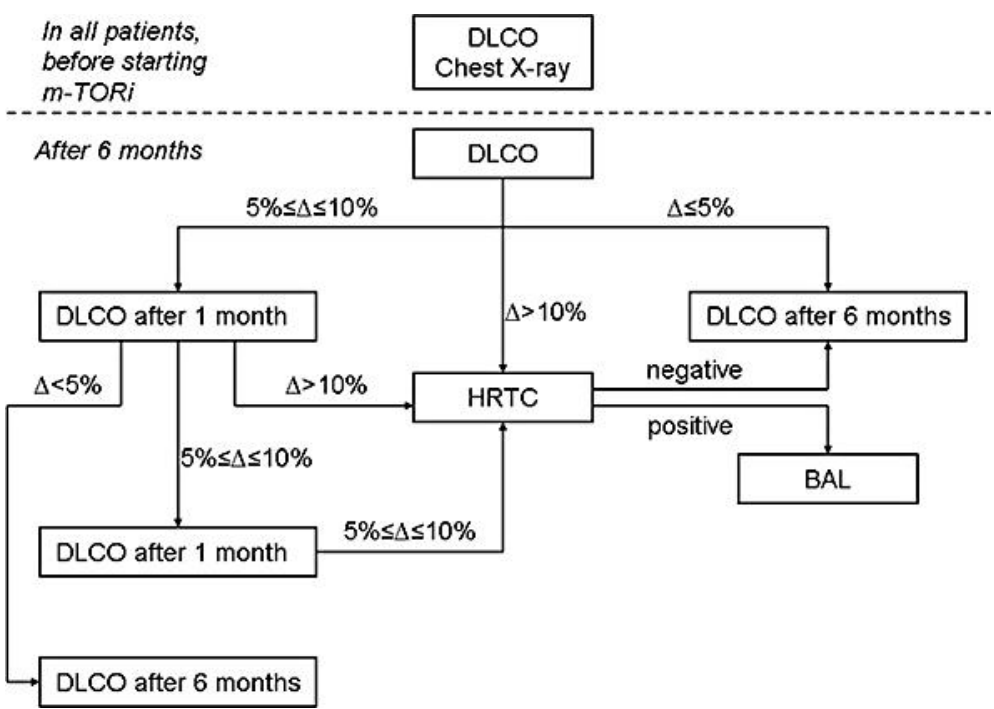

Fig. 3. Surveillance algorithm. $\Delta=$ difference (in \%) between the last 2 DLCO tests. 